\title{
Impacto econômico entre dois modelos de compras públicas: centralizado e descentralizado
}

\author{
Economic impact between two public procurement models: centralized and decentralized \\ Impacto económico entre dos modelos de contratación pública: centralizado y \\ descentralizado
}

Jamille Soares Moreira Alves ${ }^{1}$, Virgínia Angélica Silveira Reis²,

Flávio Clemente Deulefeu ${ }^{3}$ e Fernanda Gadelha Severino ${ }^{4}$.

\section{Resumo}

Esta pesquisa objetivou analisar o impacto econômico entre modelos de gestão de compra centralizada e descentralizada. Tratase de um estudo descritivo, fundamentados em manuais operacionais e dados de compras de medicamentos de instituições da administração pública, uma com modelo de

\footnotetext{
${ }^{1}$ Especialista em Fisioterapia Hospitalar pela Faculdade Integrada do Ceará - FIC, MBA em Economia e Avaliação de Tecnologia em Saúde - Hospital Alemão Oswaldo Cruz - HAOC/FIPE, Mestre em Ciências Fisiológicas pela Universidade Estadual do Ceará - UECE. Professora. Centro Universitário UniFanor. E-mail: jamille.alves@unifanor.edu.br

ORCID: https://orcid.org/0000-0003-0213-1728

${ }^{2}$ Titulo de especialista pela Sociedade Brasileira de Reumatologia, Mestre em Medicina Clínica pela Universidade Federal do Ceará UFC, Pós Graduação em Qualidade em Ambientes Hospitalares pela Escola de Saúde Publica do Estado do Ceará - ESP - CE. Diretora de Ensino e Pesquisa do Instituto de Saúde e Gestão Hospitalar. E-mail: vlopessilveira@isgh.org.br

ORCID: https://orcid.org/0000-0002-4073-9008
}

\footnotetext{
${ }^{3}$ Residência Médica em Clínica Médica e Pneumologia, Título de Especialista em Medicina Intensiva pela Associação de Medicina Intensiva Brasileira - AMIB, Pós- graduado em Gestão Hospitalar e de Organizações de Saúde pela Universidade Federal do Ceará UFC, Pós- graduado em Qualidade em Saúde e Segurança do paciente pela Fundação Oswaldo Cruz - FIOCRUZ. Diretor Presidente do Instituto de Saúde e Gestão Hospitalar. E-mail: flaviodeulefeu@isgh.org.br ORCID: https://orcid.org/0000-0003-2733-9656

${ }^{4}$ Especialista em Fisioterapia Cardio Respiratória pela Escola de Saúde Pública do Ceará - ESP- CE, Mestre em Fisioterapia pela Universidade Federal do Rio Grande do Norte - UFRN. Professora. Universidade de Fortaleza - UNIFOR. E-mail: fernandagadelha@unifor.br ORCID: https://orcid.org/0000-0001-5210-1856
}

gestão de compras centralizada (GCC) e outra com modelo de gestão de compras descentralizado (GCD), no período de 2013 a 2015. A Razão Custo-Efetividade Incremental (RCEI) foi calculada através média anual da diferença dos custos totais de cada modelo sobre diferença dos tempos operacionais. Analisou-se 14 medicamentos de maior aquisição conforme curva ABC. Quando multiplicado total adquirido por medicamento entre os modelos pelo preço médio do triênio, houve uma redução 30,23\% na GCC. A média anual da diferença do total dos custos é $\mathrm{R} \$$ 24.124.183,42 e a diferença de tempo operacional 150 dias. A RCEI calculada foi $\mathrm{R} \$ 160.827,89$ por dia, significando menos custo e maior efetividade do GCC. Com isso pode-se concluir que a GCC se mostrou mais eficiente, devido ter apresentado menor custo e menor tempo de aquisição no período analisado. 
Descritores: Organização Social; Economia da Saúde; Sistemas de Saúde; Administração Pública.

\begin{abstract}
This research aimed to analyze the economic impact between centralized and decentralized purchasing management models. This is a retrospective study, based on operational manuals and drug procurement data from public administration institutions, one with a centralized purchasing management model (GCC) and the other with a decentralized purchasing management model (GCD), in the period from 2013 to 2015 . The incremental cost-effectiveness ratio (ICR) was calculated through the annual average of the difference of the total costs of each model over the difference in operating times. Fourteen drugs with the highest acquisition were analyzed according to the $\mathrm{ABC}$ curve. When the total multiplied by medication between the models by the average price of the triennium, there was a $30.23 \%$ reduction in GCC. The annual average of the difference in total costs is $\mathrm{R} \$$ $24,124,183.42$ and the difference in operating time is 150 days. The ICR calculated $\mathrm{R} \$$ $160,827.89$ per day, meaning less cost and greater effectiveness of the GCC. With this, it can be concluded that GCC was more efficient, due to the lower cost and lower acquisition time in the analyzed period.
\end{abstract}

Keywords: Social Organization; Health Economics; Health Systems; Public Administration.

\section{Resumen}

Esta investigación objetivó analizar impacto económico entre modelos de gestión de compras centralizada y descentralizada. Se trata de un estudio retrospectivo, fundamentado en manuales operativos y datos de compras de medicamentos de instituciones de la administración pública, una con modelo de gestión de compras centralizada (GCC) y otro con modelo de gestión de compras descentralizado (GCD), en el período de 2013 a 2015. La proporción costo-efectividad incremental (RCEI) se calculó a través de la media anual de la diferencia de los costos totales de cada modelo sobre la diferencia de los tiempos operativos. Se analizaron 14 medicamentos de mayor adquisición conforme curva ABC. Cuando se multiplicó total adquirido por medicamento entre los modelos por el precio medio del trienio, hubo una reducción del 30,23\% en la GCC. La media anual de la diferencia del total de los costos R \$ 24.124.183,42 y la diferencia de tiempo operacional 150 días. La RCEI calculada R \$ 160.827,89 por día, significando menos costo y mayor efectividad del GCC. Con ello se puede concluir que la GCC se mostró más eficiente, debido a haber 
presentado menor costo y menor tiempo de adquisición en el período analizado.

Descriptores: Organización Social; Economía de la Salud; Sistemas de Salud; Administración Pública.

\section{Introdução}

O governo brasileiro tem empregado esforços em melhorar seus processos de compras. Logo, fica evidente a necessidade de se ter uma organização da gestão da cadeia de suprimento, e esta é compreendida pelos processos de coordenação dos fluxos de informações e de materiais entre fornecedores e usuários, como num sistema interligado. A conexão entre as fases do processo, na medida em que os produtos e materiais caminham em direção ao consumidor, é baseada na otimização, ou seja, na maximização do serviço ao cliente, e na redução dos custos. Para que esse processo ocorra de maneira ordeira dentro da cadeia de suprimentos, necessita-se da logística, parte importante da cadeia de suprimento, responsável pelo planejamento, implementação e controle do fluxo e estoque de bens, serviços e informações correlatas desde a origem até o ponto de consumo ${ }^{(1)}$.

As compras no setor público exigem um modelo de gestão para cadeia de suprimentos de forma integrada e sincronizada, para garantir uma eficiência na manutenção dos estoques e de novas compras de bens e serviços. A partir de uma unidade de suprimentos bem estruturada poderá haver uma redução significativa das despesas com aquisição de materiais e consequentemente uma melhor gestão dos recursos públicos ${ }^{(1)}$.

O processo de compras públicas deve seguir a Lei 8.666/93, que delibera o seguimento de licitações para aquisição de bens e serviços, garantindo o princípio constitucional da isonomia e da seleção da proposta mais eficiente, que deve ser analisada e julgada de acordo com princípios da legalidade, impessoalidade, moralidade, publicidade e eficiência, vinculação ao instrumento convocatório, julgamento objetivo. A legislação estabeleceu cinco modalidades de licitação: concorrência, tomada de preços, convite, concurso e leilão; sendo posteriormente instituída a sexta forma denominada pregão para obtenção de bens e serviços comuns por meio da Lei 10.520 de 17 de julho de $2002^{(2)}$.

As contratações realizadas pelas Organizações Sociais não se submetem ao dever de licitar, por fazerem parte do Terceiro Setor, logo, não são consideradas de administração pública direta, o que lhes permite a flexibilidade do setor privado no processo de compras e contratação de bens e serviços. Mas, como administram as unidades com recursos públicos não estão isentas da responsabilidade de respeitar o princípio da impessoalidade, de modo que suas 
contratações devem seguir o disposto em regulamento próprio, fixando regras objetivas e impessoais para o dispêndio de recursos públicos ${ }^{(3)}$.

Existem significativos desafios aos gestores públicos no planejamento do processo de aquisição de medicamentos, pois a limitação de recursos torna essencial a busca por eficiência na administração pública, visando a garantia de condições suficientes para oferta de assistência em saúde à população. Uma aquisição de medicamentos deve considerar inicialmente a seleção dos produtos (o que comprar); quando e quanto comprar (programação); e como comprar. $\mathrm{O}$ acompanhamento e avaliação dos processos são importantes para se descobrir fragilidades e proporcionar soluções dos problemas num menor tempo possível levando a um aperfeiçoamento contínuo. De maneira geral, instituições públicas têm possibilidade de utilizar dois modelos de gestão de compras: modelo de gestão centralizada (um órgão responsável pela administração de compras de várias entidades); e modelo de gestão descentralizada (cada órgão ou entidade realiza seu próprio planejamento e compras). No caso do modelo de gestão centralizada, é possível que o processo de compras seja assumido por uma organização social ${ }^{(4)}$.

O objetivo do presente trabalho é analisar diferenças de custos e prazos existentes nos dois modelos de gestão de compras utilizado no sistema de saúde no Estado do Ceará. Buscou-se avaliar o impacto orçamentário das instituições que adotassem um dos modelos, a partir de diferenças em preços pagos e quantidades adquiridas dos medicamentos de uso hospitalar com maior consumo, baseado na curva $\mathrm{ABC}$, ferramenta gerencial que ajuda na identificação dos itens de maior importância ou impacto nos gastos. E, por último, avaliar a efetividade baseada no custo através da Razão Custo-Efetividade Incremental (RCEI).

\section{Revisão da Literatura}

\section{Gestão pública em saúde}

A gestão pública tem sido alvo de reformas administrativas no contexto do setor da saúde, especialmente considerando-se tendências de gastos crescentes e recursos limitados derivados das transições demográfica e epidemiológicas no país. Novas estratégias têm sido propostas para superação de desafios na operacionalização das compras do sistema de saúde brasileiro, em busca de ganhos de eficiência. Sendo uma das alternativas adotadas a realização de parcerias com instituições privadas sem fins lucrativos ${ }^{(5)}$.

A administração direta, União, Estados, Distrito Federal e Municípios, caracterizam-se por regime de pessoal estatutário, com provimento por concurso público (CF, arts. 37 e 39; Lei 8.112/90); 
finanças regidas pela Lei de Responsabilidade Fiscal; licitações baseadas na Lei 8.666/93 e controle interno e externo, abalizados por fiscalização contábil, financeira, orçamentária, operacional e patrimonial $(\mathrm{CF}$, arts. 70 e 74). A administração direta no contexto do sistema de saúde brasileiro apresenta baixa flexibilidade, associada a dificuldades derivadas da falta de autonomia orçamentária, financeira e administrativa, tendo pouca celeridade nos processos licitatórios (compra de insumos e equipamentos) e problemas na contratação de profissionais ${ }^{(6)}$.

A proposição de diferentes modelos de gestão no setor público tem sido direcionada à redução das dificuldades enfrentadas no sistema de saúde brasileiro, se tornando presentes na prestação do serviço por meio das: autarquia, fundação, empresa pública, sociedade de economia mista, serviço social autônomo, organização social de saúde (OSS), organização da sociedade civil de interesse público (OSCIP) e as parcerias público-privada (PPP) ${ }^{(5,6)}$.

No contexto das compras públicas, modalidades de gestão centralizada e descentralizada apresentam vantagens $\mathrm{e}$ desvantagens específicas. Segundo Salgado e Fiuza ${ }^{(7)}$, o modelo de gestão centralizada favorece ganhos de escala de compras que contrapõem aos gastos de logística, economia de escopo, sinergias em licitações de múltiplos itens e evita proximidade do comprador com os fornecedores, diminuindo os riscos de corrupção; enquanto o modelo de gestão descentralizada fortalece a economia local, há maior conhecimento do comprador em relação às necessidades e costumes locais, e há redução de custos de transporte.

\section{Compras públicas em saúde}

O processo de compras públicas deve seguir preceitos previstos na Lei 8.666/93, que determina diferentes modalidades de licitação. O critério de definição da modalidade de licitação a ser adotada referese ao valor estimado, ou seja, valor das compras públicas a serem realizadas. Cada tipo de licitação apresenta valores estabelecidos, recomendações de procedimentos, definição de processo e prazos. As licitações são divididas nas seguintes modalidades: concorrência (apresentação de propostas em envelopes fechados, sendo processo de compra aberto à participação de qualquer interessado); tomada de preços (apresentação de propostas em envelopes fechados, sendo processo de compra aberto à participação de interessados previamente cadastrados); convite (apresentação de propostas em envelopes fechados, sendo processo de compra aberto somente à participação de convidados, sendo necessários, no mínimo, três participantes); pregão presencial (apresentação de propostas 
em envelopes fechados, seguida de participação em sessão pública com possibilidade de apresentação de lances ao vivo); e pregão eletrônico online (apresentação de propostas, sendo processo de compra aberto a qualquer interessado) ${ }^{(7)}$.

O pregão não estima $\mathrm{o}$ valor da contratação, sendo contrário a modalidade convite, da tomada de preços e da concorrência. A decisão pela modalidade deve ser feita considerando o tipo do bem a ser adquirido. Caso seja um processo de aquisição de bem comum, na definição legal, deve-se utilizar pregão, que é a modalidade mais empregada para aquisição de bens comuns e serviços ${ }^{(8)}$.

\section{Organizações Sociais de Saúde}

A formação de parcerias consolidadas entre Estado e Organizações da Sociedade Civil (OSC), entidades públicas não estatais, com objetivo de ampliar as políticas públicas, resultou na criação das Organizações Sociais ${ }^{(9)}$. Sendo estas parte de uma tática de flexibilização da gestão pública, compreendida como a construção de bens ou serviços públicos que não sejam atividades exclusivas do Estado, sem fins lucrativos pelas entidades da sociedade, cabendo ao Estado o acompanhamento e fiscalização de tais serviços, cuja operação é repassada a entidades qualificadas como Organizações Sociais $(\mathrm{OS})^{(10)}$. É possível identificar dois objetivos das parcerias estáveis do governo com OS: garantia de flexibilidade, autonomia e responsabilidade dos serviços e incremento no controle social ${ }^{(9)}$.

As Organizações Sociais de Saúde (OSS) também constituem parte da mesma estratégia, desenvolvida pelo Ministério da Administração Federal e Reforma do Aparelho do Estado (MARE), que organizou uma reforma jurídico normativa do setor público. O anteprojeto de lei e o decreto de regulamentação dessas afirmam que "a garantia da eficiência e a qualidade dos serviços devem ser asseguradas pela descentralização da União para os Estados e destes para os Municípios, através de parceria com a sociedade, por Contratos de Gestão"(11).

As OSS contratadas alinham e aperfeiçoam os processos estatais buscando uma eficiência e qualidade dos serviços prestados, através da maior flexibilidade gerencial em áreas de recursos humanos e materiais, focando principalmente nos resultados, na qualidade, na satisfação do usuário e na adaptação à ocorrência de mudanças na demanda por serviços de saúde (12)

\section{Métodos}

Trata-se de um estudo descritivo, por delinear o comportamento do preço dos medicamentos num determinado período de 
tempo pré-determinado, e de caráter quantitativo. Fundamentado na análise de manuais de procedimentos operacionais e dados logísticos de compras públicas de medicamentos de duas instituições ligadas à administração pública, no Estado do Ceará, uma com modelo de gestão de compras centralizada (GCC) e outra com modelo de gestão de compra descentralizado (GCD).

A análise dos gastos públicos na compra dos medicamentos sob essas duas modalidades de gestão foi baseada na comparação de preços pagos e quantidades adquiridas de itens de maior aquisição no período de 2013 a 2015, dentro do modelo de GCC, a partir da construção da curva ABC, quanto à aquisição de medicamentos, sendo comparados com os dados referentes à aquisição dos mesmos medicamentos do modelo GCD.

O levantamento dos dados foi realizado por meio da coleta de informações junto ao setor responsável pelas compras de ambos órgãos de compra no sistema de saúde cearense, que forneceram detalhes sobre fluxos operacionais, identificação dos medicamentos, quantidades adquiridas e respectivos preços unitários.

Realizou-se atualização monetária dos dados de preços e gastos referentes aos anos de 2013 e 2014 para valores de 2015, utilizando o Índice de Preços ao Consumidor Amplo (IPCA) informado pelo Instituto
Brasileiro de Geografia e Estatística (IBGE). Posteriormente, foi calculado preço médio pago de cada medicamento no período de análise. Em seguida, o preço médio foi multiplicado pela quantidade total do medicamento adquirido nos dois modelos de gestão, de forma a possibilitar estimativa do custo global de obtenção do medicamento caso a aquisição fosse realizada sob um único modelo de gestão.

Em termos de efetividade, selecionouse como variável representativa de desfecho nas compras públicas o tempo operacional (dias) do processo de aquisição, desde a solicitação da compra até a entrega do medicamento, conforme descrito nos fluxos de compras obtidos junto a representantes de cada modelo de gestão. Já na análise do custo efetividade utilizou-se a RCEI, esta foi calculada pela divisão da diferença dos custos totais de compra sob cada modelo de gestão em relação à diferença dos respectivos tempos operacionais.

\section{Resultados}

$\mathrm{Na}$ análise dos resultados, utilizou-se 14 medicamentos da curva $\mathrm{ABC}$ do triênio em questão do modelo de GCC. A Tabela 1 descreve esses itens em ordem de importância na curva com sua apresentação, o valor em real gasto com eles no triênio e sua representação percentual do montante gasto com cada medicação no período. 
Tabela 1. Curva ABC de aquisição de medicamentos sob modelo de gestão centralizada. Ceará, 2013-2015.

\begin{tabular}{|c|c|c|c|c|}
\hline Classificação & Medicamentos & Apresentação & Valor $(\mathrm{R} \$)$ & \% Participação \\
\hline 1 & Piperacilina+Tazobactama & $4,5 \mathrm{~g}$ & $5.686 .193,47$ & $13,42 \%$ \\
\hline 2 & $\begin{array}{l}\text { Enoxaparina Sódica } \\
40 \mathrm{mcg}\end{array}$ & $40 \mathrm{mcg} / 0,4 \mathrm{ml}$ & $4.327 .339,11$ & $10,21 \%$ \\
\hline 3 & Polimixina B & $500.000 \mathrm{UI}$ & $3.971 .838,27$ & $9,37 \%$ \\
\hline 4 & Solução Fisiológica & $0,9 \%$ tubo $500 \mathrm{ml}$ & $2.238 .534,99$ & $5,28 \%$ \\
\hline 5 & Heparina Sódica & $\begin{array}{l}\text { 5000UI/025 ml } \\
\text { subcutâneo }\end{array}$ & $1.967 .419,37$ & $4,64 \%$ \\
\hline 6 & $\begin{array}{l}\text { Imepenem + } \\
\text { CilastatinaSódica }\end{array}$ & $500 \mathrm{mg}$ & $1.869 .313,78$ & $4,41 \%$ \\
\hline 7 & Meropenem & $1 \mathrm{~g}$ & $1.858 .963,61$ & $4,39 \%$ \\
\hline 8 & $\begin{array}{l}\text { Enoxaparina Sódica } \\
60 \mathrm{mcg}\end{array}$ & $60 \mathrm{mcg} / 0,6 \mathrm{ml}$ & $1.818 .246,40$ & $4,29 \%$ \\
\hline 9 & Omeprazol & $40 \mathrm{mg} \mathrm{F} / \mathrm{A}$ & $1.245 .297,70$ & $2,94 \%$ \\
\hline 10 & Fentanila & $50 \mathrm{mcg} / \mathrm{ml} \mathrm{FA} 10 \mathrm{ml}$ & $1.099 .363,28$ & $2,59 \%$ \\
\hline 11 & Teicoplanina & $400 \mathrm{mg}$ & $1.095 .814,38$ & $2,59 \%$ \\
\hline 12 & Oxacilina & $500 \mathrm{mg}$ & $946.728,81$ & $2,23 \%$ \\
\hline 13 & Cefepime & $2 \mathrm{~g}$ & $833.029,10$ & $1,97 \%$ \\
\hline 14 & Ciprofloxacina & 200 mg-bolsa & $710.246,16$ & $1,68 \%$ \\
\hline
\end{tabular}

Fonte: Elaboração própria, a partir de dados coletados em pesquisa de campo.

A Tabela 2 compara os valores e quantidades do triênio entre os modelos centralizado e descentralizado, onde se observa que o modelo GCC compra em menor quantidade e com menor preço a maioria dos itens em questão. Nos anos de 2013 e 2014 se percebe em mais itens essa disparidade e em 2015 apenas em 4 itens o modelo GCD tem valor mais caros que os da GCC. 
Tabela 2. Distribuição da aquisição de medicamentos sob dois modelos de gestão pública no Estado de Ceará. Ceará, 2013-2015.

\begin{tabular}{|c|c|c|c|c|c|c|c|c|c|c|c|c|}
\hline & \multicolumn{4}{|c|}{2013} & \multicolumn{4}{|c|}{2014} & \multicolumn{4}{|c|}{2015} \\
\hline & \multicolumn{2}{|c|}{$\begin{array}{c}\text { Modelo de Gestão } \\
\text { Centralizada }\end{array}$} & \multicolumn{2}{|c|}{$\begin{array}{l}\text { Modelo de Gestão } \\
\text { Descentralizada }\end{array}$} & \multicolumn{2}{|c|}{$\begin{array}{c}\text { Modelo de Gestão } \\
\text { Centralizada }\end{array}$} & \multicolumn{2}{|c|}{$\begin{array}{c}\text { Modelo de Gestão } \\
\text { Descentralizada }\end{array}$} & \multicolumn{2}{|c|}{$\begin{array}{c}\text { Modelo de Gestão } \\
\text { Centralizada }\end{array}$} & \multicolumn{2}{|c|}{$\begin{array}{l}\text { Modelo de Gestão } \\
\text { Descentralizada }\end{array}$} \\
\hline & PU (R\$) & QUANT & PU (R\$) & QUANT & PU (R\$) & QUANT & PU (R\$) & QUANT & PU (R\$) & QUANT & PU (R\$) & QUANT \\
\hline Cefepime & 5,16 & 21.000 & 10,3 & 198.900 & 8,66 & 21.040 & 9,67 & 198.900 & 10,29 & 52.710 & 7,29 & 197.600 \\
\hline $\begin{array}{l}\text { Ciprofloxacin } \\
\text { a }\end{array}$ & 2,94 & 62.600 & 3,31 & 152.490 & 2,98 & 49.560 & 3,34 & 191.239 & 2,68 & 141.282 & 3,02 & 191.239 \\
\hline Enoxaparina & & & & & & & & & & & & \\
\hline $\begin{array}{l}\text { Sódica } \\
40 \mathrm{mcg}\end{array}$ & 18,67 & 121.200 & 15,5 & 273.390 & 16,57 & 36.000 & 16,57 & 273.390 & 15,53 & 94.520 & 15,73 & 273.390 \\
\hline Enoxaparina & & & & & & & & & & & & \\
\hline $\begin{array}{l}\text { Sódica } \\
60 \mathrm{mcg}\end{array}$ & 28,28 & 43.300 & 23,47 & 187.330 & 22,15 & 7.990 & 22,1 & 187.330 & 20,71 & 20.130 & 21,21 & 187.330 \\
\hline Fentanila & 2,94 & 129.000 & 0,88 & 474.325 & 3,3 & 91.000 & 1,76 & 255.030 & 2,19 & 191.375 & 1,61 & 449.505 \\
\hline $\begin{array}{l}\text { Heparina } \\
\text { Sódica }\end{array}$ & 8,19 & 148.500 & 6,23 & 155.320 & 5,5 & 65.000 & 5,91 & 257.195 & 3,59 & 109.700 & 3,62 & 190.320 \\
\hline Imepenem + & & & & & & & & & & & & \\
\hline $\begin{array}{l}\text { Cilastatina } \\
\text { Sódica }\end{array}$ & 11,1 & 56.200 & 12,41 & 483.430 & 11,05 & 21.000 & 13,85 & 191.780 & 24,94 & 40.644 & 12,54 & 191.780 \\
\hline
\end{tabular}




\begin{tabular}{lrrrr|rrrrrrrr} 
Meropenem & 22,2 & 25.650 & 19,42 & 130.160 & 19,67 & 17.000 & 20,77 & 238.500 & 17,38 & 54.958 & 14,58 & 269.700 \\
Omeprazol & 4,97 & 116.000 & 4,31 & 309.400 & 4,73 & 45.625 & 3,92 & 219.520 & 4,29 & 105.504 & 3,93 & 251.155 \\
Oxacilina & 0,97 & 433.200 & 2,86 & 105.430 & 2,21 & 82.300 & 2,68 & 483.430 & 3,18 & 109.028 & 2,43 & 483.430 \\
Piperacilina+ & 8,36 & 143.700 & 32,71 & 264.920 & 29,77 & 62.230 & 35,7 & 209.000 & 27,54 & 95.580 & 27,78 & 309.400 \\
Tazobactama & & & & & & & & & & \\
Polimixina B & 59,3 & 22.000 & 64,84 & 78.260 & 62,42 & 29.050 & 34,38 & 97.110 & 61 & 14.000 & 21,26 & 114.400 \\
Solução & 1,48 & 283.360 & 1,52 & 187.330 & 1,66 & 218.500 & 1,4 & 2.981 .400 & 1,94 & 750.586 & 1,94 & 1.527 .070 \\
Fisiológica & & & & & & & & & & & & \\
Teicoplanina & 34,67 & 7.100 & 29,21 & 498.184 & 37,01 & 18.310 & 33,36 & 66.800 & 34,4 & 5.000 & 28,43 & 83.540 \\
\hline
\end{tabular}

Legenda: PU (R\$): preço unitário em real, QUANT: quantidade

Fonte: Elaboração própria, a partir de dados coletados em pesquisa de campo. 
Ao se calcular o preço médio dos medicamentos em ambos os modelos de gestão e somar as quantidades compradas, o gasto global do sistema de saúde poderia apresentar uma redução de $\mathrm{R} \$ 72.372 .550,26(30,23 \%)$ caso a totalidade das compras fosse realizada no modelo de GCC, em comparação ao modelo GCD no período (Tabela 3).

A efetividade, representada pelo tempo operacional, foi considerada pelo tempo total do processo de compra de cada modelo de gestão, sendo de 30 dias no modelo GCC e 180 dias no modelo GCD (Figura 1).

Tabela 3. Preço médio e quantidade adquirida dos medicamentos nos modelos de gestão centralizada e descentralizada. Ceará, 2013-2015.

\begin{tabular}{|c|c|c|c|c|c|}
\hline \multirow{2}{*}{ Medicamento } & $\begin{array}{l}\text { Quantidade } \\
\text { total }\end{array}$ & \multicolumn{2}{|c|}{$\begin{array}{l}\text { Preço } \\
\text { Médio }\end{array}$} & \multirow{2}{*}{$\begin{array}{l}\text { Quantidade total } \\
\text { a preços GCC }\end{array}$} & \multirow{2}{*}{$\begin{array}{c}\text { Quantidade total } \\
\text { a preços GCD }\end{array}$} \\
\hline & $\begin{array}{l}\text { GCC } \\
+ \text { GCD }\end{array}$ & GCC & GCD & & \\
\hline Cefepime & 690.150 & 8,79 & 13,6 & $6.066 .418,50$ & $9.386 .040,00$ \\
\hline Ciprofloxacina & 788.410 & 2,8 & 5 & $2.207 .548,00$ & $3.942 .050,00$ \\
\hline $\begin{array}{l}\text { Enoxaparina Sódica } \\
40 \mathrm{mcg}\end{array}$ & 1.071 .890 & 17,19 & 23,9 & $18.425 .789,10$ & $25.618 .171,00$ \\
\hline $\begin{array}{l}\text { Enoxaparina Sódica } \\
60 \mathrm{mcg}\end{array}$ & 633.410 & 25,46 & 33,39 & $16.126 .618,60$ & 21.149.559,90 \\
\hline Fentanila & 1.590 .235 & 2,67 & 2,18 & $4.245 .927,45$ & $3.466 .712,30$ \\
\hline Heparina Sódica & 926.035 & 6,09 & 7,7 & $5.639 .553,15$ & $7.130 .469,50$ \\
\hline $\begin{array}{l}\text { Imepenem + } \\
\text { CilastatinaSódica }\end{array}$ & 984.834 & 15,86 & 16,38 & $15.619 .467,24$ & 16.131.580,92 \\
\hline Meropenem & 735.968 & 19,05 & 30,96 & $14.020 .190,40$ & $22.785 .569,28$ \\
\hline Omeprazol & 1.047 .204 & 4,66 & 6,01 & $4.879 .970,64$ & 6.293.696,04 \\
\hline Oxacilina & 1.696 .818 & 1,52 & 4,71 & $2.579 .163,36$ & $7.992 .012,78$ \\
\hline Piperacilina+Tazobactama & 1.084 .830 & 18,86 & 52,16 & $20.459 .893,80$ & $56.584 .732,80$ \\
\hline Polimixina B & 354.820 & 61,06 & 61,83 & $21.665 .309,20$ & $21.938 .520,60$ \\
\hline Solução Fisiológica & 5.948 .246 & 1,79 & 2,34 & $10.647 .360,34$ & $13.918 .895,64$ \\
\hline
\end{tabular}


Teicoplanina

$678.93436,03 \quad 33,9$

24.461.992,02

$23.015 .862,60$

Gasto global

167.045.201,80

239.353.873,36

Figura 1. Fluxo de compras nos modelos de gestão centralizada e descentralizada. Ceará,

2013-2015 


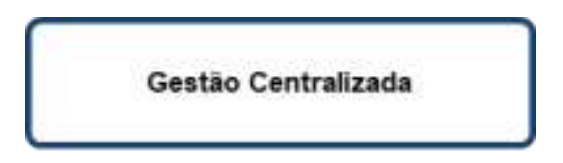

Recebe solicitação de compra

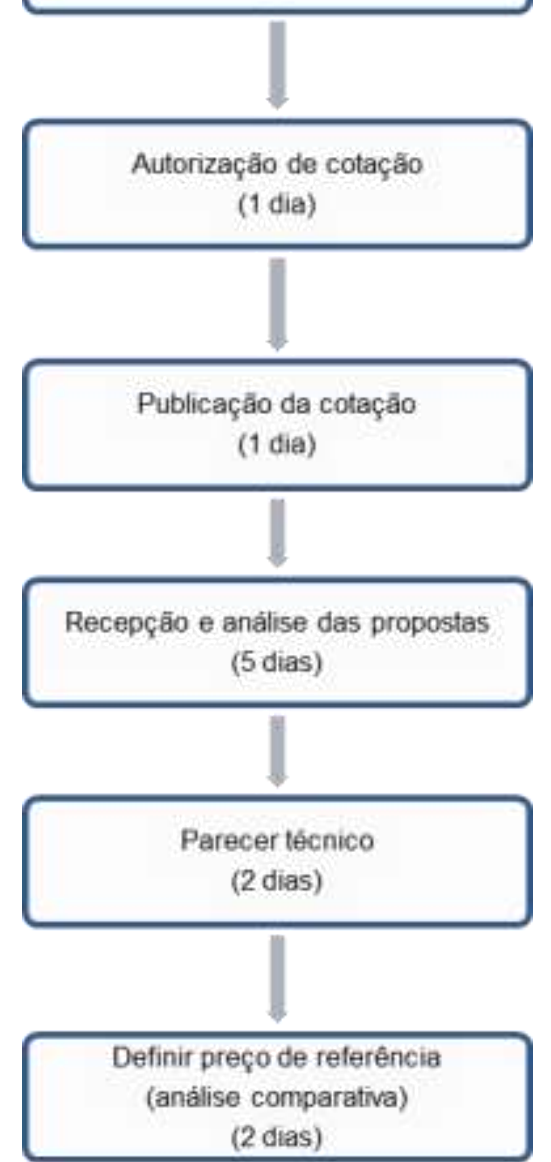

Negociaçäo

Critério de escolha

(menor preça/melhor técnica)

Conferência de habilitaçāo técnica, fiscal e legal

(2 dias)

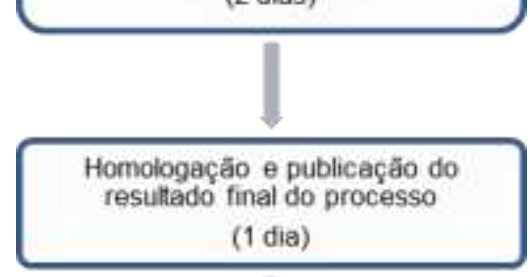

Processo de compra finalizado para emissăo de $\mathrm{AF}$ para o núcleo de planejamento (1 dia)

\section{Gestão Descentralizada}

Recebe solicitação de compra

Abrir processo licitatório (NAl)

(2 dias)

零

Avaliação do descritivo (NATS)

(2 dias)

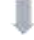

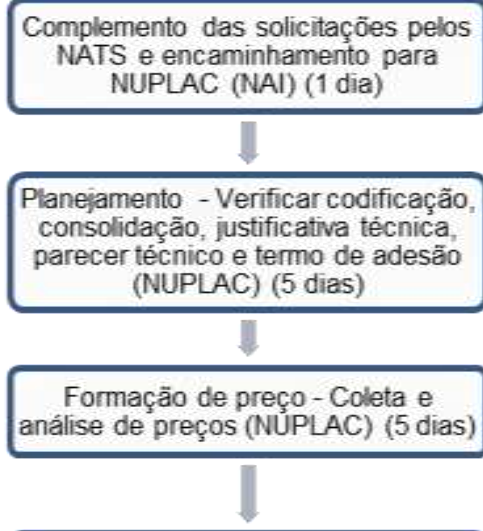

Execução - Solicitar demandas, fazer instruções e acompanhamento do processo (NUPLAC) (5 dias)

Emitir parecer jurídico (ASJUR) (5 dias)

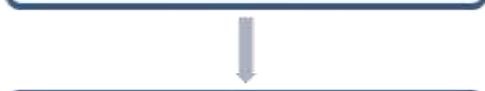

Fase externa - Entre a marcação e a realização do certame - Pregão (PGE) (132 dias)

Análise das amostras - Envio pelos representantes e analsar amostra (NATS) (7 dias)

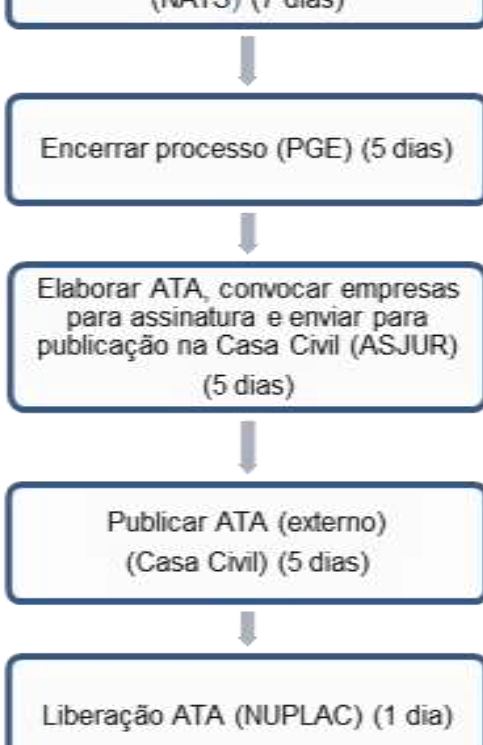


Legenda: NAI: Núcleo de Aquisição e Insumos, NATS: Núcleo de Avaliação de Tecnologia em Saúde, NUPLAC: Núcleo de Planejamento de Compras, PGE: Procuradoria Geral do Estado, ASJUR: Assessoria Jurídica Fonte: Elaboração própria, a partir de dados coletados.

A diferença global de custos observada foi $\mathrm{R} \$ 72.308 .671,56$ e a diferença de tempo operacional foi 150 dias, ambas em favor da gestão centralizada de compras.

A razão custo-efetividade incremental (RCEI), calculada utilizando a diferença dos custos globais sob cada modelo de gestão em relação ao período global de processo de aquisição, foi $\mathrm{R} \$ 482.057,81$ por dia no período de 2013 a 2015, que representa uma razão positiva e uma estratégia dominante, considerando menor custo e maior efetividade do modelo de gestão centralizada.

\section{Discussão}

O principal achado do presente estudo foi a diferença na comparação dos valores e no tempo decorrido na aquisição de medicamentos pelo modelo GCC em relação ao modelo GCD, observando-se a razão custo-efetividade que determinou cenário favorável para a aquisição na modalidade de GCC.

As OSS são uma realidade na saúde do Brasil e desde a sua implantação vêm fomentando debates político e social. De modo geral, quando analisadas sob a perspectiva da gestão orçamentária e financeira, os benefícios do modelo são $\operatorname{expressivos}^{(9)}$. O que pode ser demonstrado com os achados do estudo em questão, onde as compras da GCC, modelo da OSS, conseguiram ser mais econômicas e mais rápidas em relação às compras $\mathrm{GCD}$, que foi realizada por unidades de administração pública direta. Garantindo assim maior eficácia e eficiência nos processos de aquisições de medicamentos, que são um grande desafio nas unidades de saúde.

A sociedade e a mídia cobram com mais veemência instituições públicas mais eficientes e focadas em seus propósitos e com isto os gestores são incentivados a buscar ferramentas de gestão mais atualizadas, enaltecendo a transparência e sendo cientificamente reconhecidas como eficientes levando a uma valorização dos recursos públicos e satisfação do cidadão-cliente ${ }^{(13)}$. Através dos dados coletados, observa-se que a transparência está presente em ambos os modelos de gestão de compra, uma vez que as instituições apresentaram seus dados nos sites e disponibilizaram seus fluxos de trabalho para a realização da pesquisa em questão.

A centralização objetiva atingir a eficiência na aquisição, através da otimização do processo de compra pela redução de atividades sobrepostas, levando a economias de escala. Com a possibilidade de diminuição dos custos unitários do produto, devido ao 
aumento da capacidade de negociação, aumento do volume, padronização das compras, prazos determinados e entregas fracionadas costumam apresentar benefícios como abastecimento constante, diminuição dos estoques e nos custos de armazenamento, garantia de medicamentos de acordo com os prazos de validade e planejamento financeiro $^{(14)}$.

A economicidade é um princípio a ser levado em conta no momento da compra. Prover os insumos necessários para as atividades fins de um negócio garantindo a qualidade demandada, com preços e prazos competitivos, é um desafio ${ }^{(13)}$.

No estudo em questão pode ser observado, no período analisado, que nem sempre comprar em larga escala gerava a economicidade esperada. O modelo GCD adquiriu maiores quantidades do que o modelo GCC, mas ainda assim tinham valores unitários maiores, levando o modelo GCC comprar mais barato e em menor quantidade. Acredita-se que o fato do processo de compra do modelo GCC ser mais rápido, levando a um processo de pagamento aos fornecedores num tempo mais curto seria um dos motivos do GCC conseguir preços melhores, além disso, a OSS em questão encontrava-se num período de expansão das unidades geridas por ela e, consequentemente, tem aumentado o volume de compra de medicamentos para suprir essa demanda, o que também poderia ter influenciado nesses valores.
Um estudo realizado no interior do Ceará, Banabuiú também comparou a compra centralizada, realizada por ele, com quatros cidades de Pernambuco com características semelhantes à cidade cearense que pratica compra descentralizada. E tiveram como resultado a GCC como a opção mais econômica para a aquisição das medicações da atenção primária ${ }^{(15)}$.

$\mathrm{Na}$ tentativa de entender as causas, identifica-se que a central de compras deve ser dinâmica e garantir a equidade, segurança, acessibilidade e qualidade dos insumos adquiridos. Planejamento e rigor na aquisição dos medicamentos com um sistema de informação integrado permitindo a partilha e comunicação em rede entre a central de compras e as unidades, fomentando uma política de proximidade, é de suma importância para o sucesso da compra, sendo, portanto, um ponto a ser levado em conta ${ }^{(14)}$.

Entendendo que muito dos preços de compra desfavoráveis se dão devido à pressão por resultados imediatos, culminando com o aumento do custo e gerando a ineficiência do serviço prestado, pois se utiliza com maior frequência das compras diretas (com dispensa e inexigibilidade de licitação) e modalidades de licitação mais rápidas e menos burocráticas, como a carta-convite. No entanto, esses procedimentos, quando utilizados sem planejamento adequado, são antieconômicos, pois geram fracionamento de despesa, são compras emergenciais e isso leva 
a valores mais elevados do que os negociados nas compras padrão e apresentam um risco maior de irregularidades devido a suas fragilidades $^{(16)}$.

Outro ponto a ser abordado é a questão do tempo gasto por cada um dos modelos de gestão de compra, a efetividade de uma compra perpassa pelo tempo gasto entre a solicitação e a chegada do insumo na unidade. O modelo de GCC apresentou tempo operacional inferior, correspondendo a uma redução de $83,33 \%$ no tempo do processo de aquisição de medicamentos em relação ao processo de compras no modelo de GCD.

Esse achado leva a uma reflexão em relação ao processos de compras públicas: burocracia, com tempos regimentais que os tornam complexos, devido o processo licitatório que tem um arcabouço normativolegal próprio ${ }^{(13)}$. Faz-se necessária revisão de processos potencialmente morosos de forma amparada pela legislação. No que tange à gestão de suprimentos, o bom desenvolvimento organizacional perpassa pela revisão dos processos internos de gestão, pela organização de um modelo de gestão capaz de propor mudanças e implantá-las, assim como buscar por transparência na ação dos entes participantes $^{(17)}$.

Independente do modelo de compra a ser adotado é necessário ter como pressuposto atingir um desempenho mais eficaz da administração em relação às compras públicas, levando em consideração o bom atendimento às necessidades do estado (governança), dos cidadãos (finalidade social), às empresas (promoção do desenvolvimento) e à sociedade como um $\operatorname{todo}^{(18)}$.

A gestão de compras públicas pode promover responsabilidade social de várias formas, incluindo-se possibilidade de estímulo são surgimento de vagas de emprego e renda no país, amparo aos discriminados, conservação ambiental, inclusão educacional e diminuição da desigualdade.

\section{Conclusão}

O modelo de gestão de compra centralizada, praticado pela OSS, demonstrou um impacto econômico em relação à economia financeira e a redução do tempo entre a abertura da solicitação e a chegada do medicamento nas unidades.

No que diz respeito à economia do tempo gasto entre a solicitação e a entrega dos medicamentos, o modelo centralizado gasta em média 30 dias versus 180 dias do modelo descentralizado. O modelo de gestão de compra descentralizada, administração direta, é regido pela Lei 8.666/93, enquanto o modelo centralizado, OSS, tem normas a seguir que estão previstas no contrato de gestão, porém menos burocráticas do que as normas da lei acima citada. Outro ponto a ser analisado em relação ao tempo, se dá na relação entre comprador-fornecedor, como o 
modelo GCC leva um tempo menor, os fornecedores acabam recebendo os seus pagamentos mais rápido e isso levou esse modelo a comprar com preços melhores do que o GCD, apesar deste último comprar em maiores quantidades.

E, por último, a análise demonstra a efetividade da compra através RCEI, onde o modelo de GCC mostrou-se mais efetivo devido apresentar menores custos e tempo operacional.

$\mathrm{O}$ artigo em questão não esgota esse assunto, mais estudos devem ser desenvolvidos a fim de garantir a melhor utilização do dinheiro público, focando na eficiência e transparência. 


\section{Referências}

1. Tridapalli pj, fernandes e, machado wv. Gestão da cadeia de suprimento do setor público: uma alternativa para controle de gastos correntes no brasil. Revista de administração pública [internet]. 2011[acesso em 2019 jan 20];45(2),401-33. Disponível em: http://www.scielo.br/scielo.php?script =sci_arttext\&pid=s0034-

$76122011000200006 \& \operatorname{lng}=\mathrm{en} \& \mathrm{nrm}=\mathrm{i}$ so

2. Brasil. Lei $\mathrm{n}^{0} 10.520$, de 17 julho de 2002. Institui, no âmbito da união, estados, distrito federal e municípios, nos termos do art. 37, inciso xxi, da constituição federal, modalidade de licitação denominada pregão, para aquisição de bens e serviços comuns, e dá outras providências. Brasília: diário oficial [da] república federativa do brasil, [2018]. Disponível em: http://www.presidencia.gov.br/ccivil_ 03/leis/2002/110520.htm. Acesso em: 20 jan 2018.

3. Brasil. Presidência da república casa civil. Lei $\mathrm{n}^{0}$ 9.637.dispõe sobre a qualificação de entidades como organizações sociais, a criação do programa nacional de publicização, a extinção dos órgãos e entidades que menciona e a absorção de suas atividades por organizações sociais, e dá outras providências. Brasília: presidência da república casa civil, 15 maio de 1998. Disponível em: http://www.planalto.gov.br/ccivil_03/1 eis/19637.htm. Acesso em: 20 jan 2018.

4. Bevilacqua $\mathrm{g}$, farias $\mathrm{mr}$, blatt $\mathrm{cr}$. Aquisição de medicamentos genéricos em município de médio porte. Rev saúde pública[internet]. 2011 [acesso em 2019 mar 08]; 45(3),583-9. Disponível em: http://www.scielo.br/scielo.php?script $=$ sci_arttext $\&$ pid $=$ s003489102011000300017

5. Andreazzi mfs, bravo mis. Privatização da gestão e organizações Rev. Gestão \& Saúde (Brasília) Vol. 10, n. 03, Set. 2019. sociais na atenção à saúde. Trab. Educ. Saúde. [internet]. 2014[acesso em 2019 mar 08.];12(3), 499-518. Disponível em http://www.scielo.br/scielo.php?script =sci_arttext $\&$ pid $=$ s1981$77462014000300499 \& \operatorname{lng}=$ pt\&nrm $=$ is $\mathrm{O}$

6. Nogueira, rp. O desenvolvimento federativo do sus e as novas modalidades institucionais de gerência das unidades assistenciais. In: santos, nr dos; amarante, pdc (org.). Gestão pública e relação público privado na saúde. Rio de janeiro: cebes. [internet]. 2010 [acesso em 2019 mar 15.]; 24-47. Disponível em: http://idisa.org.br/img/file/gc-2010-rllivro\%20cebes-2011.pdf

7. Salgado lh, fiuza, eps. Marcos regulatórios no brasil: aperfeiçoando a qualidade regulatória. Rio de janeiro, rj: ipea. 2015.

8. Freitas $\mathrm{m}$, maldonado jms de $\mathrm{v}$. $\mathrm{O}$ pregão eletrônico e as contratações de serviços contínuos. Rev. Adm. Pública[internet]. 2013 [acesso em 2019 mar 08.]; 47(5), 1265-81. Disponível em: http://www.scielo.br/scielo.php?pid=s 0034-

$76122013000500009 \&$ script $=$ sci_abst ract

9. Sano h,abrucio fl. Promessas e resultados da nova gestão pública no brasil: o caso das organizações sociais de saúde em são paulo. Revista de administração de empresas -rae [internet]. 2008 [acesso em 2018jan12.]; 48(3), 64-80. Disponível em:http://www.scielo.br/scielo.php?sc ript=sci_arttext\&pid=s0034$75902008000300007 \& \operatorname{lng}=$ en \&nrm=i so

10. Soares gbet al.. Organizações sociais de saúde (oss): privatização da gestão de serviços de saúde ou solução gerencial para o sus?.revista eletrônica gestão \& saúde. [internet]. 2016 [acesso em 2019 mar 24.]; 2(7), 828- 
50. Disponível

em:http://periodicos.unb.br/index.php/ rgs/article/view/3547

11. Barbosa nb, elias pem. As organizações sociais de saúde como forma de gestão público/privado. Ciência \& saúde coletiva.[internet]. 2010 [acesso em 2018 mar 24.]; 15(5), 2483-95. Disponível em: http://www.scielo.br/scielo.php?pid=s $1413-$

$81232010000500023 \&$ script $=$ sci_abst ract\&tlng=pt

12. Tibério aa, souza em, sarti fm. Considerações sobre avaliação de estabelecimentos de saúde sob gestão de oss: o caso do hospital geral do grajaú. Saúde soc.[internet]. 2010 [acesso em 2018jan15.]; 19(3), 557$68 . \quad$ Disponível em: http://www.scielo.br/scielo.php?script =sci_arttext\&pid=s0104$12902010000300008 \& \operatorname{lng}=\mathrm{en} \& \mathrm{nrm}=\mathrm{i}$ so

13. Nishiyama ma, lima mva,ensslin 1 , chavesl. Modelo multicritério para avaliação de desempenho: um estudo de caso para gestão de compras no setor público. Revista de ciências da administração.[internet]. 2017 [acesso em 2018jan 28.];19(47), 9-28.

Disponível em:

https://periodicos.ufsc.br/index.php/ad $\mathrm{m} /$ article/view/2175-

8077.2017v19n47p9

14. Aperta $\mathrm{j}$, borges a, cadilha d, dimas $\mathrm{f}$, dinis c, feio jet al.. Compras centralizadas na saúde. Revportfarmacoter..[internet]. 2016 [acesso em 2019 jan 15.];7(4), 214-20, disponível em: http://revista.farmacoterapia.pt/index.p hp/rpf/article/view/97.
15. Barreira filho md, torres $\mathrm{kb}$, ribeiro $\mathrm{db}$, prado rmda s, sobrinho jls. Avaliação do modelo de compra centralizada de medicamentos: estudo de caso da assistência farmacêutica básica. Boletim informativo geum. 2015; 6(1), 80-9.

16. Arraes jp da s. A reestruturação do setor de compras da universidade de brasília: a implantação de uma nova estrutura de compras, visando à melhoria dos processos, à redução das compras diretas e à otimização dos recursos públicos. Future studiesresearchjournal.[internet]. 2017 [acesso em 2019 mar 24.]; 9(2),16787.disponível em:https://www.revistafuture.org/fsrj/ article/view/293/401.

17. Carneiro d, resende p. Processo de compra de medicamentos no setor público: uma abordagem qualitativa sobre inovação organizacional. In: congresso ibero -americano de investigação qualitativa, 2017, salamanca. Atas ciaiq2017. [internet]. 2017 [acesso em 2019 mar 24.]; disponível

em:https://proceedings.ciaiq.org/index .php/ciaiq2017/article/view/1143/1109

18. Araújo rsr, gomes cs. Análise comparativa da vantajosidade entre compras governamentais centralizadas e descentralizadas, brasília. In: iii congresso consad de gestão pública, 3 , 2010, brasília.anais [...] Brasília: conselho nacional de secretários de administração, 2010. P. 1-22.

Disponível em: http://banco.consad.org.br/handle/123 456789/116. Acesso

em: 10 fev 2018

\section{Participação dos autores:}

Alves JSM trabalhou na concepção teórica, coleta de dados, análise estatística e elaboração e redação final do texto.

Reis VAS redação final do texto.

Deulefeu FC redação final do texto. 
Severino FG trabalhou na concepção teórica, elaboração e redação final do texto.

Recebido: 30.07.2019

Revisado: 09.08.2019

Aprovado: 20.09.2019 